\title{
Determinação de tempo médio mínimo necessário para a determinação de um padrão emocional na entoação frasal $^{9}$
}

\author{
Lucas Negri
}

\section{Introduçâo}

De acordo com António Damásio (DAMÁSIO, 1996), as emoções são configurações fisiológicas que têm influência direta no funcionamento de outras áreas do cérebro, inclusive as responsáveis pela tomada de decisões e pelo uso da razão abstrata. Segundo esse autor, informações provenientes do corpo têm influência direta no pensamento, como atestam as deficiências de pessoas com problemas nos córtices cerebrais somatossensoriais do hemisfério direito, parte do cérebro responsável por receber as sinapses vindas do corpo e repassar suas interpretações a outras regiões do cérebro. Pessoas com essa deficiência apresentam problemas de raciocínio, na tomada de decisões e também com emoções e sentimentos (DAMÁSIO, 1996, p.90-96).

O trabalho de Skinner (1935) é pioneiro na análise da relação entre situação emocional e entoação vocálica. Nesse trabalho, o autor capta, na simples pronúncia de um "a" alongado, depois de o falante estar sujeito a incentivos emocionais, padrões de

\footnotetext{
9 SIMPÓSIO INTERNACIONAL DE INICIAÇÃO CIENTÍFICA/USP - SIICUSP, 23. São Paulo, 2015.
} 
ondas sonoras diferentes para pessoas sujeitas a estímulos tristes e a estímulos alegres.

Outra referência considerada para este trabalho foi o texto Os reveladores da mentira (ALMEIDA JR., 1940), sobre a captação de situações emocionais a partir de respostas corpóreas. Ainda, para além desses trabalhos, a relação entre performance vocálica e emoções se estende em vasta bibliografia.

Para a análise de entoação frasal no presente trabalho, baseamo-nos principalmente nos trabalhos de Ferreira Netto (2006, 2008a). Sendo assim, consideramos entoação como "uma sequência de tons, iguais ou diferentes, produzidos pela voz durante a fala" (FERREIRA NETTO, 2013). Ferreira Netto, desenvolvedor do programa ExProsodia, propôs que a entoação da fala pode ser decomposta em componentes estruturadores finalização (F) e sustentação (S) - e semântico-funcionais foco/ênfase (E).

De acordo com Ferreira Netto (2006; 2008a) e Peres e seus colegas $(2009 ; 2011)$, o ato de falar é composto de uma série de tensões e relaxamentos físicos. Os momentos de tensão, ou de esforço, são considerados os momentos de sustentação (S) da fala, em que se produz um tom de voz numa determinada frequência a que chamamos F0. A média aritmética, no tempo, dos F0 produzidos determina o tom médio ideal (TM) da fala. Os momentos de relaxamento no ato de fala produzem uma declinação pontual de aproximadamente 7 semitons, nos pontos denominados de finalização $(\mathrm{F})$, quando a continuação do ato de fala demanda a retomada da tensão de sustentação, em novo F0, que decairá 7 semitons até o próximo ponto de finalização F. Sendo assim, o ato de falar acontece numa sucessão de retomadas de tensão, a que chamamos "unidades básicas de entonação" (Units of Base of Intonation - UBI) que compõem, sucessivamente e em conjunto, a entoação frasal. 
Ainda de acordo com Ferreira Netto e outros (2014), é possível identificar padrões entoacionais para as emoções de cólera, embora a diferença entre entoações neutras e tristes não se verifique. No entanto, entoações coléricas não se diferem de acordo com o gênero, mas as neutras e tristes sim, são diferentes entre homens e mulheres. Tal análise foi feita com a leitura do programa ExProsodia.

Além disso, Ferreira Netto e seus colegas (FERREIRA NETTO; MARTINS; VIEIRA; SPANGHERO, 2014) identificaram um padrão entoacional denominado Simulacrum of Neutral Intonation, um padrão entoacional que marca uma espécie de "acordo" entre o que se diz e o que se sente. A análise com o programa ExProsodia capta uma divergência entre falas "sinceras" e falas interpretadas, acusando o desacordo emocional entre o que o corpo realmente está sentindo e o que se está tentando passar através da voz. Essa captação, além de servir como possível detector de mentiras, também pode ser usada para identificar a presença de psicopatia em pacientes psiquiátricos.

Determinar a medida temporal mínima que nos fornece UBIs suficientes para a análise emocional da fala possibilitará desenvolvermos o software para o processamento simultâneo da captação, para análise em tempo real da entoação frasal.

\section{Metodologia}

Foram selecionadas 6 gravações, retiradas da internet, sendo 3 correspondentes a pessoas com raiva (dois homens e uma mulher) e 3 a pessoas tristes (duas mulheres e um homem). Esse procedimento tem a vantagem de as pessoas não saberem que suas falas seriam objeto de pesquisa sobre a pronúncia, ainda que as gravações utilizadas tenham sido feitas conscientemente, isto é, as pessoas sabiam que suas falas estavam sendo gravadas - sendo uma gravação um discurso no 
Congresso Nacional, outra a filmagem amadora de um prisioneiro e, as outras quatro, entrevistas.

Cada gravação foi dividida em 5 partes de igual duração, e foi selecionado um momento aleatório em cada uma dessas partes. A partir de cada um desses momentos, foram feitos recortes de aproximadamente 0,5 segundo, 1 segundo, 1,5 segundo, 2 segundos e 3 segundos na gravação - utilizando o software Speech Filing System ${ }^{\circledR}$ - totalizando 25 recortes por gravação. Foram utilizados recortes aproximados porque o programa Speech Filing System ${ }^{\circledR}$ não permite recortes em qualquer medida, modificando as medidas cabíveis de acordo com a extensão total do arquivo. Como padronização, optou-se por manter a aproximação sempre para cima ou sempre para baixo na medida de recorte buscada na gravação. Por exemplo, na busca por recortes de 1 segundo numa gravação, o programa apenas autorizava recortes de 1,0072 segundo. Foi então mantido o recorte de 1,0072 segundo para todos os trechos de 1 segundo buscados na gravação.

Ainda com esse mesmo software foram retirados, de cada um dos 150 trechos selecionados, uma análise de frequência e uma de intensidade, a serem lidas pelo software ExProsodia para determinar quantos momentos UBI são captáveis em cada um desses intervalos de tempo - e todos os dados e resultados foram catalogados.

O software ExProsodia oferece os seguintes parâmetros relativos à frequência:

- menor_FO_UBI -- menor frequência alcançada em F0

- maior_FO_UBI - maior frequência alcançada em FO

- extensao_FO - extensão vertical média de F0

- dp_FO_UBI - desvio padrão de F0

- skew_FO_UB - assimetria (skew) de FO

- mediana_FO_UBI - mediana de FO

- CV_FO_UBI - coeficiente de variação de FO 
- kurt_FO_UBI - curtose de FO

- num_UBI - quantidade de UBIs no trecho

- UBI_final - frequência da UBI final

- TM-mUBI - diferença entre o TM e o menor valor de UBI (a rigor é

TM - menor_FO_UBI)

- menor_TM - menor frequência alcançada no tom médio

- maior_TM - maior frequência alcançada no tom médio

- TM - tom médio

- dp_TM - desvio padrão de TM

- skew_TM - assimetria (skew) de TM

- mediana_TM - mediana de TM

- kurt_TM - curtose de TM

- Cv_TM - coeficiente de variação de TM

- menor_FEpos_UBI - menor frequência positiva alcançada de foco/ênfase

- maior_FEpos_UBI - maior frequência positiva alcançada de foco/ênfase

- media_FEpos_UBI - frequência média de foco/ênfase positivo

- dp_FEpos_UBI - desvio padrão de foco/ênfase positivo

- skew_FEpos_UBI - assimetria (skew) de foco/ênfase positivo

- extensao_FEpos_UBI-mediana de foco/ênfase positivo

- CV_FEpos_UBI - coeficiente de variação de foco/ênfase positivo

- kurt_FEpos_UBI-curtose de foco/ênfase positivo

- menor_FEneg_UBI - menor frequência negativa alcançada de foco/ênfase

-maior_FEneg_UBI - maior frequência negativa alcançada de foco/ênfase

- media_FEneg_UBI - frequência média de foco/ênfase negativo

- dp_FEneg_UBI - desvio padrão de foco/ênfase negativo

- skew_FEneg_UBI - assimetria (skew) de foco/ênfase negativo

- extensao_FEneg_UBI-mediana de foco/ênfase negativo

- Cv_FEneg_UBI - coeficiente de variação de foco/ênfase negativo

- kurt_FEneg_UBI - curtose de foco/ênfase negativo

Dos 5 recortes com a mesma medida de tempo de cada gravação, por exemplo, dos cinco recortes de 0,5 segundo, foi calculada a média de valores para cada um desses parâmetros determinados pelo programa ExProsodia. 
Montamos uma matriz com todos esses valores médios encontrados em cada recorte de tempo de cada gravação e fizemos um cálculo estatístico de correlação de dados, com o software Kyplot.

O objetivo do trabalho era, a partir dessa leitura, determinar qual recorte temporal é o mínimo necessário para captar parâmetros UBI para a análise entoacional. Pelo cálculo estatístico, a correlação de dados que acusasse $r^{2} \leq 0,4$, com $P \leq 0,05$, apontaria uma diferença significativa dos dados, o que representaria uma diferença significativa da leitura entre uma medida de tempo e outra.

Com o mesmo software, Kyplot, também realizamos análises estatísticas de Cluster, na busca de parâmetros que acusassem a especificidade de uma ou outra situação de fala marcada nas gravações.

Com essas análises poderíamos determinar com quantas UBI já é possível considerar um trecho de fala que corresponde ou pode ser comparado a trechos anteriores para a identificação de uma determinada emoção geral ou padrão normal.

\section{Resultado final}

A análise com o programa ExProsodia mostrou as seguintes médias de captura de UBIs:

\begin{tabular}{|l|c|c|c|}
\hline & Bravo & Triste & Geral \\
\hline 0,5 segundo & 3,2 & 3,2 & 3,2 \\
\hline 1 segundo & 5,7 & 4,9 & 5,3 \\
\hline 1,5 segundo & 7,6 & 6,1 & 6,8 \\
\hline 2 segundos & 9,6 & 8,9 & 9,2 \\
\hline 3 segundos & 13,6 & 12,7 & 13,1 \\
\hline
\end{tabular}


Tabela1. Quantidade média de UBIs captadas pelo ExProsodia

Já a correlação de dados acusou que, para a captação dos parâmetros pelo programa ExProsodia, é indiferente a captação de 0,5 segundo, 1 segundo, 1,5 segundo, 2 ou 3 segundos. Nenhum dos dados apresentou $P \leq 0,05$ e $r^{2} \leq 0,04$. Ou seja, tanto faz se captarmos 0,5 segundo ou 3 segundos de voz, a quantidade de dados oferecidos é relativamente a mesma para a determinação das emoções.

A análise estatística de Cluster, no entanto, acusou dados mais interessantes.

Dendrogram

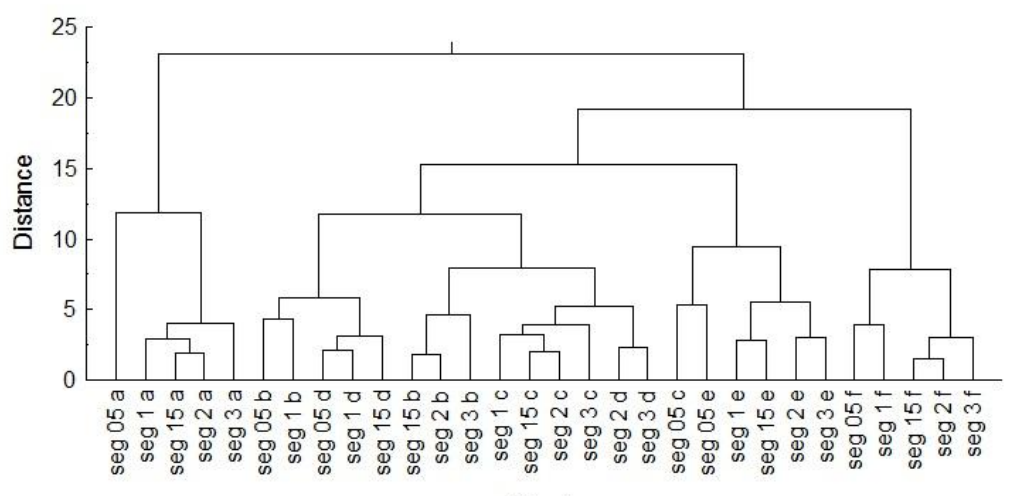

Objects

Figura 1. Dendograma das médias gerais

As letras de $a$ a $f$ no gráfico designam as gravações da seguinte forma:

a - CidinhaCamposBrava (mulher brava)

b - FuzileiroBravo (homem bravo)

c - MataMaeFacadas (homem bravo)

$\mathrm{d}$ - EsposaMilitarMorto (mulher triste) 


\section{e - FilhaChoraAMae (mulher triste) \\ $f$ - TioFalaDoSobrinho (homem triste)}

Podemos observar, pela imagem, que os dados das gravações $a$ e $f$ ficaram isolados nos extremos do dendograma. De fato, são as gravações que apresentam os extremos em relação aos dados analisados: a gravação CidinhaCamposBrava, de uma mulher brava, apresenta os valores mais altos de TM e de F0; e a gravação TioFalaDoSobrinho, de um homem triste, apresenta os mais baixos; estando em acordo com a pesquisa de Skinner (1935) assim como com FERREIRANETTO et Alii. (2013), que indica valores mais altos desses parâmetros para o sexo feminino e o sentimento de cólera, e valores mais baixos para o sexo masculino e o sentimento de tristeza.

Apenas, no entanto, as outras gravações se confundem um pouco na proximidade de seus parâmetros.

\section{Análises}

A confusão entre as gravações $b, c, d$ e $e$ indica uma incapacidade de identificar objetivamente mulheres tristes e homens bravos, provavelmente devido a estes apresentarem F0 e TM mais elevados em relação ao seu TM neutro, que é mais baixo, e, aquelas, F0 e TM mais rebaixados em relação ao seu TM neutro, que é mais alto, o que aproxima seus FO e TM nas emoções em questão.

Essa confusão se desfaz quando analisamos as gravações b e d isoladamente, como se pode ver no dendograma seguinte. Isso provavelmente porque, sem precisar se preocupar com a comparação com valores extremos, a comparação entre os valores mais próximos fica mais sutil, acurada; ou então, porque a mulher triste da gravação $d$ apresenta momentos de indignação na sua fala, o que a aproximaria do sentimento de cólera, tornando a separação de dados mais clara por sua 
entoação aumentar a pontos mais altos do que é o usual para homens.

\section{Dendrogram}

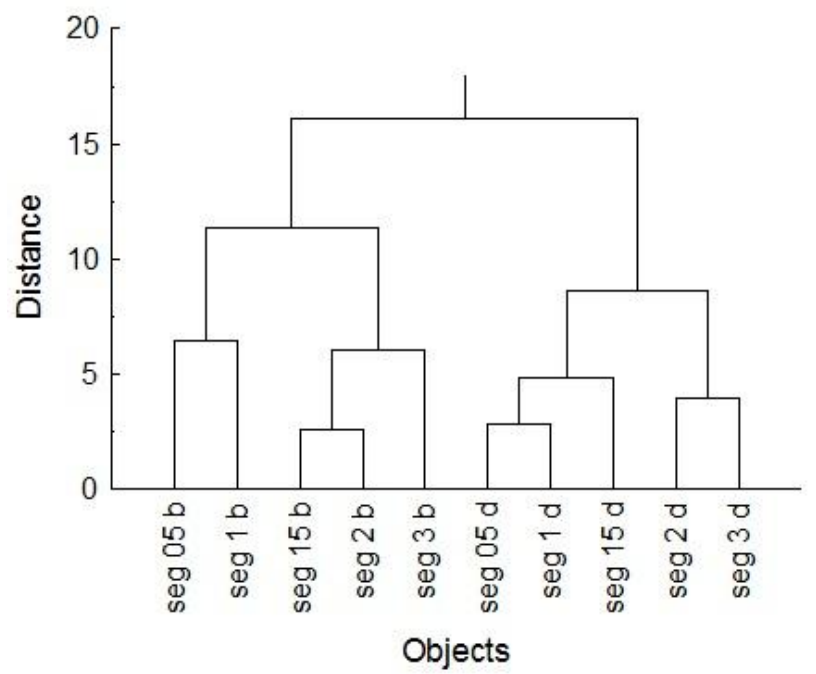

Figura 2. Dendograma gravações $b$ e $d$

Já mesmo a análise isolada entre $c$ e $d$ ou entre $c$ e $e$ não separou bem os dados. Mesmo essa análise isolada não apresenta apontamento significativo sobre a melhor medida de tempo, como podemos ver nos dendogramas seguintes, em que o primeiro indica uma distância maior entre os trechos de 0,5 segundo do que entre os trechos de 2 ou 3 segundos, e o segundo indica uma distância maior entre os trechos de 2 e 3 segundos, mesmo para a separação entre os sexos. 


\section{Dendrogram}

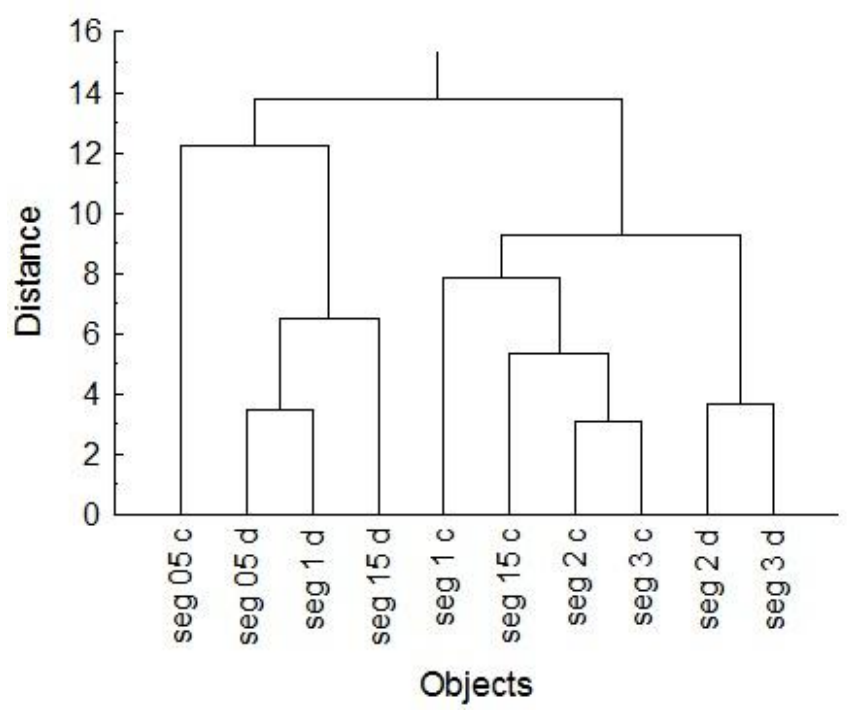

Figura 3. Dendograma gravações $c$ e $d$ 


\section{Dendrogram}

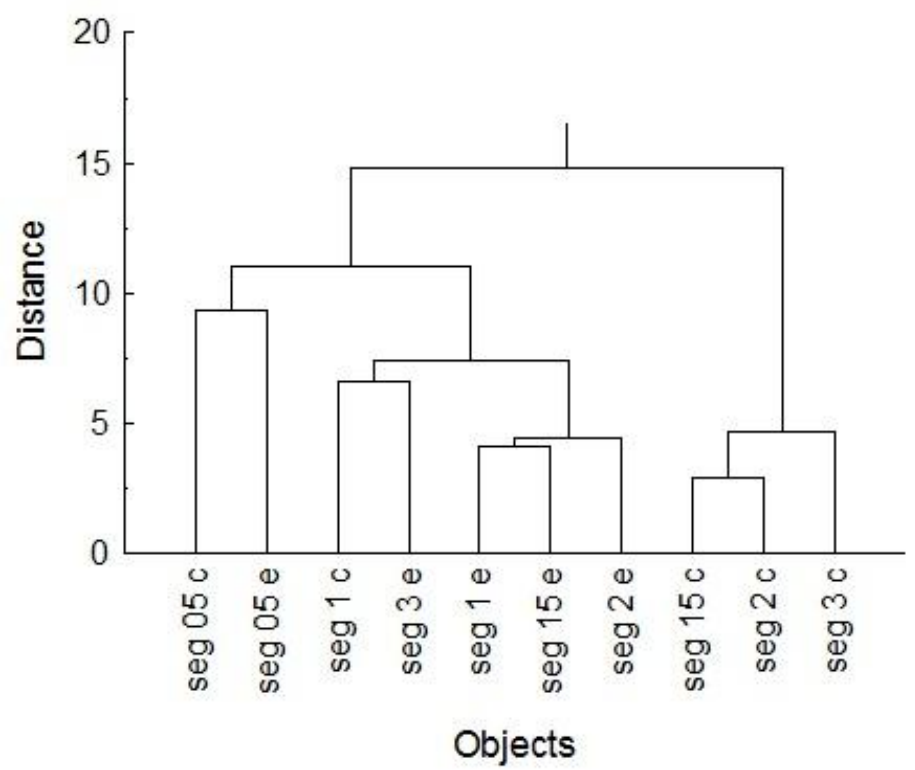

Figura 4. Dendograma gravações $c$ e $e$

Devido ao fato de os parâmetros para homens bravos e mulheres tristes se confundirem, parece haver a necessidade de uma hierarquização de valores na análise entoacional da fala: primeiro seria necessário estabelecer uma separação entre os gêneros e, depois, a captação da emoção. Sem saber se a fala analisada é do gênero masculino ou do gênero feminino, o software é incapaz, com as medidas de tempo analisadas, de indicar tratar-se de um homem bravo ou de uma mulher triste, embora seja capaz de identificar, para qualquer uma das medidas de tempo utilizadas, o homem triste ou a mulher brava.

Em Ferreira Netto e outros (2008b, 2009), é sugerido que o foco/ênfase é uma categoria capaz de diferenciar os gêneros. 
Talvez seja possível, em um trabalho futuro, conferir se esse parâmetro pode contribuir para a identificação mais precisa da entoação, sem a necessidade à parte da hierarquização supracitada.

Também sugerimos que a pesquisa com outros parâmetros oferecidos pelo ExProsodia, como duração e intensidade, não considerados nesta pesquisa, possam oferecer complementação aos resultados.

\section{Conclusão}

A correlação dos dados mostrou que não há diferença significativa nos parâmetros adquiridos com trechos de 0,5 segundo, 1 segundo, 1,5 segundo, 2 ou 3 segundos.

A análise estatística de Cluster mostrou que, para qualquer medida de tempo dentre as analisadas, utilizando os parâmetros de frequência e tom médio, é possível identificar o homem triste e a mulher brava, mas a identificação de homens bravos e mulheres tristes se confunde. Isso sugere a necessidade de pesquisar outros parâmetros que possibilitem distingui-los, como por exemplo a duração ou o foco/ênfase, ou então uma hierarquização na pesquisa, que parta de uma definição do gênero a priori para então identificar a emoção na entoação frasal. 